\title{
A NOTE ON TRIGONOMETRIC MATRICES
}

\section{GARRET J. ETGEN}

Introduction. Let $Q(x)$ be an $n \times n$ symmetric matrix of continuous functions on $X: 0 \leqq x<\infty$ and let the pair of $n \times n$ matrices $\{S(x), C(x)\}$ denote the solution of the second order matrix differential system

$$
\begin{gathered}
Y^{\prime}=Q(x) \cdot Z, \quad Z^{\prime}=-Q(x) \cdot Y \\
Y(0)=0, \quad Z(0)=E(\text { the } n \times n \text { identity matrix })
\end{gathered}
$$

on $X$. This pair of matrices was introduced by J. H. Barrett [2] in order to study the behavior of the solutions of the second order selfadjoint matrix differential system

$$
\left[P(x) \cdot Y^{\prime}\right]^{\prime}+F(x) \cdot Y=0 .
$$

Also, W. T. Reid [5] used the solution pair of (1) in order to study the properties of the solutions of the second order matrix differential system

$$
Y^{\prime}=K(x) \cdot Z, \quad Z^{\prime}=G(x) \cdot Y .
$$

More recently, the author [3] established that the pair $\{S(x), C(x)\}$ has many of the properties of the sine and cosine functions. In particular, $S(x)$ and $C(x)$ satisfy identities on $X$ which are the matrix analogues of the elementary trigonometric identities and, in the event $Q(x)$ is positive definite on $X$, the pair $\{S(x), C(x)\}$ has oscillatory behavior which is analogous to the oscillatory behavior of $\left\{\sin \int_{0}^{x} q(t) d t, \cos \int_{0}^{x} q(t) d t\right\}$, where $q(x)$ is a positive, continuous function on $X$. In this paper we establish that there exists a positive number $p$ such that if $Q(x)$ is positive definite on $X, a$ is any nonnegative number and $b, b>a$, has the property $\int_{a}^{b} \operatorname{tr} Q(x) d x \geqq p$, then at least one of $S(x)$ and $C(x)$ has a singularity on $a \leqq x \leqq b$. Note that, in the case $n=1, p=\pi / 2$. This property, together with the results of $[3, \S 1]$, completes the analogy between the oscillatory behavior of the solution pair of (1), where $Q(x)$ is positive definite on $X$, and the oscillatory behavior of the pair $\left\{\sin \int_{0}^{x} q(t) d t, \cos \int_{0}^{x} q(t) d t\right\}, q(x)>0$ on $X$. An application of this result is discussed in $\S 3$.

1. A double angle formula. Barrett [2, Theorem 1.1, p. 511] has

Presented to the Society, September 1, 1966; received by the editors May 4, 1966. 
shown that the solution pair $\{S(x), C(x)\}$ of (1) satisfies the following identities on $X$ :

$$
\begin{gathered}
C(x) \cdot C^{*}(x)+S(x) \cdot S^{*}(x)=E=C^{*}(x) \cdot C(x)+S^{*}(x) \cdot S(x), \\
C(x) \cdot S^{*}(x)=S(x) \cdot C^{*}(x), \quad C^{*}(x) \cdot S(x)=S^{*}(x) \cdot C(x),
\end{gathered}
$$

where $\left({ }^{*}\right)$ denotes the transpose of the indicated square matrix.

We use these identities to establish the following "double angle" formula.

THEOREM 1.1. Let the pair of $n \times n$ matrices $\{\Phi(x), \Psi(x)\}$ denote the solution of the matrix differential system:

$$
\begin{gathered}
Y^{\prime}=Q(x) \cdot Z+Z \cdot Q(x), \quad Z^{\prime}=-Q(x) \cdot Y-Y \cdot Q(x), \\
Y(0)=0, \quad Z(0)=E,
\end{gathered}
$$

on $X$. Then, the following identities hold:

$$
\Phi(x)=2 S(x) \cdot C^{*}(x), \quad \Psi(x)=C(x) \cdot C^{*}(x)-S(x) \cdot S^{*}(x),
$$

(cf. $\sin [2 x]$ and $\cos [2 x])$,

$$
\begin{gathered}
\Phi^{2}(x)+\Psi^{2}(x)=E, \\
\Phi(x) \cdot \Psi(x)=\Psi(x) \cdot \Phi(x) .
\end{gathered}
$$

Moreover, each of $\Phi(x)$ and $\Psi(x)$ is symmetric on $X$.

Proof. Put $G=2 S C^{*}$ and $H=C C^{*}-S S^{*}$. Then,

$$
G^{\prime}=2 S\left(C^{*}\right)^{\prime}+2 S^{\prime} C^{*}=-2 S S^{*} Q+2 Q C C^{*} .
$$

But, since $Q C C^{*}+Q S S^{*}=C C^{*} Q+S S^{*} Q$, we have $Q C C^{*}-S S^{*} Q$ $=C C^{*} Q-Q S S^{*}$. Thus

$$
G^{\prime}=Q C C^{*}-S S^{*} Q+C C^{*} Q-Q S S^{*}=Q H+H Q .
$$

Similarly, it is verified that $H^{\prime}=-Q G-G Q$. Since $G(0)=0$ and $H(0)=E$, it follows that $G(x)=\Phi(x)$ and $H(x)=\Psi(x)$. Thus (7) is established.

Identities (8) and (9) can be verified directly using (4) and (5). Finally, it is clear that $\Phi(x)$ and $\Psi(x)$ are both symmetric.

2. Oscillatory behavior. In the case $n=1, S(x)=\sin \int_{0}^{x} Q, C(x)$ $=\cos \int_{0}^{x} Q, \Phi(x)=\sin 2 \int_{0}^{x} Q$ and $\Psi(x)=\cos 2 \int_{0}^{x} Q$, and if $Q(x)>0$ on $X$, then the oscillatory behavior of $S, C, \Phi$, and $\Psi$ is determined. Consequently, for $n$ an arbitrary positive integer we assume, in the work which follows, that the continuous, symmetric $n \times n$ matrix $Q(x)$ is positive definite on $X$. 
Definition. A solution $Y(x)$ of either (1) or (6) is said to be oscillatory on $X$ if for each nonnegative number $b$ there is a number $c, c>b$, such that $|Y(c)|=0 . Y(x)$ is said to be nonoscillatory on $X$ if it is not oscillatory.

Using Theorem 1.1 and the techniques and results of $[3, \S 1]$, the following oscillatory properties of $\Phi(x)$ and $\Psi(x)$ are easily verified.

TheOREM A. If $b, b>0$, is the first number such that $|\Psi(b)|=0$, then $\Phi(x)$ is nonsingular on $0<x<b$.

THEOREM B. If $a \leqq x \leqq b$ is a subinterval of $X$ such that $\Phi(x)$ is nonsingular for $a \leqq x \leqq b$, then $\Psi(x)$ has at most $n$ singularities on this interval. Similarly, if $\Psi(x)$ is nonsingular on $c \leqq x \leqq d$, then $\Phi(x)$ has at most $n$ singularities on $c \leqq x \leqq d$.

Theorem C. $\Phi(x)$ and $\Psi(x)$ cannot be identically singular on any subinterval of $X$. Moreover, the singularities of $\Phi(x)$ and $\Psi(x)$ cannot have a finite limit point.

Theorem D (Separation Theorem). If $a$ and $b$ are consecutive singularities of $\Phi(x)$, then $\Psi(x)$ has at most $n$ singularities on $a \leqq x \leqq b$. Similarly, if $c$ and $d$ are consecutive singularities of $\Psi(x)$, then $\Phi(x)$ has at most $n$ singularities on $c \leqq x \leqq d$.

THEOREM E. If $\int_{0}^{\infty} \operatorname{tr} Q(x) d x=\infty$ and $a$ is any nonnegative number, then both $\Phi(x)$ and $\Psi(x)$ have infinitely many singularities on $a \leqq x<\infty$.

Theorem F. A necessary and sufficient condition that $\Phi(x)$ and $\Psi(x)$ be nonoscillatory on $X$ is $\int_{0}^{\infty} \operatorname{tr} Q(x) d x<\infty$.

For each $x$ on $X$, let $s_{j}(x)$ and $c_{j}(x), j=1,2, \cdots, n$, denote the $n$ characteristic roots of $\Phi(x)$ and $\Psi(x)$ respectively. Since each of $\Phi(x)$ and $\Psi(x)$ is continuous and symmetric on $X$, the functions $s_{j}(x)$ and $c_{j}(x), j=1,2, \cdots, n$, are real and continuous on $X$. We note that the zeros of these functions correspond to the singularities of $\Phi(x)$ and $\Psi(x)$.

Lemma 2.1. Fix any $x$ on $X$. If $s$ is a characteristic root of $\Phi(x)$ with associated characteristic vector $\alpha$, then $\alpha$ is also a characteristic vector of $\Psi(x)$ and, if $c$ is the characteristic root of $\Psi(x)$ associated with $\alpha$, then $s^{2}+c^{2}=1$.

Proof. Fix $x$ on $X$ and let $s$ and $\alpha$ be as described in the hypothesis. From (8), $\alpha \Phi^{2}+\alpha \Psi^{2}=\alpha$. Thus $\alpha \Psi^{2}=\left(1-s^{2}\right) \alpha$. Consequently, $\alpha$ is a characteristic vector of $\Psi$ associated with either $c=\left[1-s^{2}\right]^{1 / 2}$ or $c=-\left[1-s^{2}\right]^{1 / 2}$. Clearly, $s^{2}+c^{2}=1$. 
Let $P(x)$ be the $n \times n$ matrix defined by the equation $P(x)=\Psi(x)$ $+i \Phi(x)$ on $X, i^{2}=-1$ (c.f. $e^{i x}=\cos x+i \sin x$ ).

TheOREM 2.1. The matrix $P(x)$ has the following properties on $X$ :

(a) $P(x)$ is the solution of the matrix differential system

$$
W^{\prime}=i[Q(x) W+W Q(x)], \quad W(0)=E .
$$

(b) $P(x)$ is unitary, i.e., $P(x) \bar{P}^{*}(x)=E$, where $\bar{P}^{*}$ denotes the conjugate transpose of $P$.

(c) $|P(x)|=\exp \left\{2 i \cdot \int_{0}^{x} \operatorname{tr} Q(t) d t\right\}$.

(d) Fix any $x$ on $X$. If $s$ and $c$ are characteristic roots of $\Phi(x)$ and $\Psi(x)$ respectively associated with the characteristic vector $\alpha$, then $\alpha$ is a characteristic vector of $P(x)$ and $c+i \cdot s$ is the associated characteristic root.

Proof. Properties (a) and (b) can be verified directly.

(c) According to Reid [4], the solution of $K^{\prime}=B(x) \cdot K+K \cdot B^{*}(x)$ is $K=J(x) \cdot M \cdot J^{*}(x)$ where $M$ is a constant matrix and $J(x)$ is the solution of $J^{\prime}=B(x) \cdot J, J(0)=E$. Since $Q(x)$ is symmetric, we have $P(x)=J(x) \cdot M \cdot J^{*}(x)$ where $J^{\prime}=i Q(x) \cdot J$ and $J(0)=E$. Evaluating at $x=0$ yields $M=E$. Thus $|P(x)|=|J(x)|^{2}$. However, by Abel's Identity, $|J(x)|=\exp \left\{i \cdot \int_{0}^{x} \operatorname{tr} Q(t) d t\right\}$. Therefore, $|P(x)|$ $=\exp \left\{2 i \cdot \int_{0}^{x} \operatorname{tr} Q(t) d t\right\}$.

(d) Fix an $x$ on $X$ and let $s$ and $c$ be characteristic roots of $\Phi$ and $\Psi$ respectively associated with the characteristic vector $\alpha$. Then $\alpha P=\alpha \Psi+i \alpha \Phi=(c+i s) \alpha$ and hence $\alpha$ is a characteristic vector of $P$ with associated characteristic root $c+i s$. Thus the theorem is established.

For each $x$ on $X$, let $p_{j}(x), j=1,2, \cdots, n$, denote the $n$ characteristic roots of $P(x)$. Since $P(x)$ is continuous and unitary on $X$, the $n$ functions $p_{j}(x)$ are continuous and, for each $x$ on $X$ and each integer $j, 1 \leqq j \leqq n, p_{j}(x)$ is a complex number with norm 1 .

Now, using arguments similar to that of Atkinson [1, Appendix V] and the author [3], we show that:

THEOREM 2.2. The characteristic roots of $P(x)$ move around the unit circle in the positive direction as $x$ increases on $X$.

Proof. Fix any $x_{0}$ on $X$ and let $\theta$ be a constant such that $e^{i \theta}$ is not a characteristic root of $P\left(x_{0}\right)$. Then, there is a positive number $\epsilon$ such that $e^{i \theta}$ is not a characteristic root of $P(x)$ for $x$ on $X_{\epsilon}: x_{0}-\epsilon$ $<x<x_{0}+\epsilon$.

Lemma 2.2. If $A(x)=i\left[e^{i \theta} E+P(x)\right]\left[e^{i \theta} E-P(x)\right]^{-1}$ on $X_{\epsilon}$, then $A(x)$ is hermitian and $A^{\prime}(x)$ is positive definite on $X_{\mathrm{\epsilon}}$. 
Proof.

$$
\begin{aligned}
\bar{A}^{*} & =-i\left[e^{-i \theta} E+\bar{P}^{*}\right]\left[e^{-i \theta} E-\bar{P}^{*}\right]^{-1} \\
& =-i\left[e^{-i \theta} E+\bar{P}^{*}\right] e^{i \theta} P\left[P-e^{i \theta} E\right]^{-1} \\
& =-i\left[P+e^{i \theta} E\right]\left[P-e^{i \theta} E\right]^{-1} \\
& =i\left[e^{i \theta} E+P\right]\left[e^{i \theta} E-P\right]^{-1}=A .
\end{aligned}
$$

For notational convenience, put $V=e^{i \theta} E-P$. Now, $A(x)$ can be written $A=2 i e^{i \theta} V^{-1}-i E$. Calculating $A^{\prime}$, we have

$$
A^{\prime}=2 i e^{i \theta} V^{-1} P^{\prime} V^{-1}=-2 e^{i \theta}\left[V^{-1} Q P V^{-1}+V^{-1} P Q V^{-1}\right] .
$$

But it is readily verified that $P V^{-1}=V^{-1} P=-e^{-i \theta}\left(\bar{V}^{*}\right)^{-1}$. Thus

$$
A^{\prime}=2 V^{-1} Q\left(\bar{V}^{*}\right)^{-1}+2\left(\bar{V}^{*}\right)^{-1} Q V^{-1}
$$

and, since $Q(x)$ is positive definite, it follows that $A^{\prime}$ is positive definite on $X_{\epsilon}$.

Now for each $x$ on $X_{\epsilon}$ let $a_{j}(x), j=1,2, \cdots, n$, denote the $n$ real characteristic roots of $A(x)$. Since $A(x)$ is differentiable and $A^{\prime}(x)$ is positive definite, the functions $a_{j}(x)$ are continuous and increasing on $X_{\epsilon}$.

Fix any $x$ on $X_{\epsilon}$ and let $p$ be a characteristic root of $P(x)$ with corresponding characteristic vector $\alpha$. Then,

$$
\alpha A=i \alpha\left[e^{i \theta} E+P\right] \cdot\left[e^{i \theta} E-P\right]^{-1}=i\left[\left(e^{i \theta}+p\right) /\left(e^{i \theta}-p\right)\right] \alpha,
$$

that is, $i\left(e^{i \theta}+p\right) /\left(e^{i \theta}-p\right)$ is a characteristic root of $A(x)$ with corresponding characteristic vector $\alpha$.

Consider the mapping $w=i\left(e^{i \theta}+z\right) /\left(e^{i \theta}-z\right)$. This mapping takes the positively described real axis into the positively described unit circle. Consequently, as $w$ increases, $z$ moves around the unit circle in the positive sense. Now, since the characteristic root functions $a_{j}(x), j=1,2, \cdots, n$, of $A(x)$ are increasing on $X_{\epsilon}$, it follows that the characteristic root functions $p_{j}(x), j=1,2, \cdots, n$, of $P(x)$ move positively on the unit circle for $x_{0}-\epsilon<x<x_{0}+\epsilon$.

Since $x_{0}$ was any point on $X$, it follows that the characteristic roots of $P(x)$ move positively on the unit circle as $x$ increases on $X$ and the theorem is established.

Since $P(0)=E, p_{j}(0)=1, j=1,2, \cdots, n$. For each positive in teger $j, 1 \leqq j \leqq n$, and each $x$ on $X$, let $w_{j}(x)$ denote the argument of the complex number $p_{j}(x)$. Define $w_{j}(0)=0, j=1,2, \cdots, n$, and assume that the functions $w_{j}(x)$ are continued as continuous functions as $x$ increases on $X$. Since the characteristic roots of $P(x)$ move in the positive direction on the unit circle as $x$ increases, the continuous functions $w_{j}(x)$ are increasing on $X$. 
TheOREM 2.3. If $a$ is any nonnegative number and $b, b>a$, has the property $\int_{a}^{b} \operatorname{tr} Q(x) d x \geqq n \pi / 2$, then both $\Phi(x)$ and $\Psi(x)$ have at least one singularity on $a \leqq x \leqq b$.

Proof. Consider $P(x)=\Psi(x)+i \Phi(x)$. By Theorem 2.1 (c), $|P|$ $=\exp \left\{2 i \cdot \int_{0}^{x} \operatorname{tr} Q\right\}$. On the other hand,

$$
|P(x)|=\prod_{j=1}^{n} p_{j}(x)=\prod_{j=1}^{n} \exp \left[i w_{j}(x)\right]=\exp \left[i \cdot \sum_{j=1}^{n} w_{j}(x)\right] .
$$

Consequently,

$$
\sum_{j=1}^{n} w_{j}(x)=2 \int_{0}^{x} \operatorname{tr} Q(t) d t+2 k \pi
$$

for some integer $k$. But evaluating at $x=0$ gives $k=0$. Thus

$$
\sum_{j=1}^{n} w_{j}(x)=2 \int_{0}^{x} \operatorname{tr} Q(t) d t .
$$

Now, since the functions $w_{j}(x)$ are positive, continuous and increasing on $X, \int_{a}^{b} \operatorname{tr} Q(t) d t \geqq n \pi / 2$ implies that at least one of the functions $w_{1}, w_{2}, \cdots, w_{n}$, say $w_{j}$, increased by an amount greater than or equal to $\pi$ on $a \leqq x \leqq b$. But $w_{j}(x)$ is the argument of $p_{j}(x)$ and $p_{j}(x)$ can be written $p_{j}=c+i \cdot s$, where $c$ and $s$ are characteristic roots of $\Psi(x)$ and $\Phi(x)$ respectively. Since $w_{j}(x)$ increased by an amount greater than or equal to $\pi$, each of $s(x)$ and $c(x)$ had at least one zero on $a \leqq x \leqq b$ and, consequently, each of $\Phi(x)$ and $\Psi(x)$ had at least one singularity on $a \leqq x \leqq b$.

Corollary 2.3.1. If $\{S(x), C(x)\}$ is the solution of (1) with $Q(x)$ positive definite on $X$, if $a$ is any nonnegative number and if $b, b>a$, has the property $\int_{a}^{b} \operatorname{tr} Q(x) d x \geqq n \pi / 2$, then at least one of $S(x)$ and $C(x)$ has a singularity on $a \leqq x \leqq b$.

Proof. This corollary is a consequence of identity (7) and Theorem 2.3.

3. An application. Consider the second order matrix differential systems (2), (3) and the nonlinear system

$$
Y^{\prime}=K[x ; Y ; Z] \cdot Z, \quad Z^{\prime}=G[x ; Y ; Z] \cdot Y
$$

defined in $[3, \S 2]$ on the interval $X$.

In [1, Chapter 10] and [3], it was necessary to explicitly calculate the characteristic roots of $P(x), F(x), K(x)$ and $G(x)$ in order to give estimates of oscillation of solutions. Now, as a consequence of Corollary 2.3.1, we can give estimates of oscillation of solutions of certain 
systems having the form of either (2), (3) or (10) which do not depend on calculating the characteristic roots of the matrices.

Let the pair of $n \times n$ matrices $\{Y(x), Z(x)\}$ denote the solution of either (2), (3) or (10) on $X$ such that $Y(0)=0, Z(0)=E$. Then, as established in [2], [3] and [5], $Y(x)$ and $Z(x)$ can be written $Y=S^{*}(x) \cdot R(x), Z(x)=C^{*}(x) \cdot R(x)$, where $R(x)$ is nonsingular on $X$ and $\{S(x), C(x)\}$ is the solution of (1) with $Q(x)=C P^{-1} C^{*}+S F S^{*}$ for $\{Y, Z\}$ the solution of $(2)$ and $Q(x)=C K C^{*}-S G S^{*}$ for $\{Y, Z\}$ the solution of either (3) or (10).

Now, in system (2), assume that each of $P$ and $F$ is positive definite and either $P^{-1}-F$ or $F-P^{-1}$ is nonnegative definite for each $x$ on $X$; in systems (3) and (10), assume that each of $K$ and $-G$ is positive definite and either $K+G$ or $-G-K$ is nonnegative definite for each $x$ on $X$. Then it is readily verified that $Q$ is positive definite on $X$ and

$$
\min \left[\operatorname{tr} P^{-1}, \operatorname{tr} F\right] \leqq \operatorname{tr} Q \leqq \max \left[\operatorname{tr} P^{-1}, \operatorname{tr} F\right]
$$

in system (2) and, in systems (3) and (10),

$$
\min [\operatorname{tr} K, \operatorname{tr}(-G)] \leqq \operatorname{tr} Q \leqq \max [\operatorname{tr} K, \operatorname{tr}(-G)] .
$$

THEOREM 3.1. If, in system (2), $a$ is any nonnegative number and $b$, $b>a$, has the property: $\min \left[\int_{a}^{b} \operatorname{tr} P^{-1}, \int_{a}^{b} \operatorname{tr} F\right] \geqq n \pi / 2$ or if, in system (3) or (10), $a$ is any nonnegative number and $b, b>a$, has the property: $\min \left[\int_{a}^{b} \operatorname{tr} K, \int_{a}^{b} \operatorname{tr}(-G)\right] \geqq n \pi / 2$, then at least one of $Y(x)$ and $Z(x)$ has a singularity on $a \leqq x \leqq b$.

\section{BIBLIOGRAPHY}

1. F. V. Atkinson, Discrete and continuous boundary problems, Academic Press, New York, 1964.

2. J. H. Barrett, A Prüfer transformation for matrix differential equations, Proc. Amer. Math. Soc. 8 (1957), 510-517.

3. G. J. Etgen, Oscillatory properties of certain nonlinear matrix differential systems of second order, Trans. Amer. Math. Soc. 122 (1966), 289-310.

4. W. T. Reid, A matrix differential equation of the Riccati type, Amer. J. Math. 68 (1946), 237-246.

5. - A Prüfer transformation for differential systems, Pacific J. Math. 8 (1958), 575-584.

6. W. M. Whyburn, Existence and oscillation theorems for nonlinear differential equations of second order, Trans. Amer. Math. Soc. 30 (1928), 848-854.

Headquarters, National Aeronautics and Space Administration, WashINGTON, D.C. 